\title{
Article
}

\section{A Novel Approach for the Detection of Developing Thunderstorm Cells}

\author{
Richard Müller * (D), Stephane Haussler, Matthias Jerg and Dirk Heizenreder \\ German Weather Service, Frankfurter Str 135, 63067 Offenbach, Germany; Stephane.Haussler@dwd.de (S.H.); \\ Matthias.Jerg@dwd.de (M.J.); Dirk Heizenreder@dwd.de (D.H.) \\ * Correspondence: Richard.Mueller@dwd.de; Tel.: +49-(0)69-8062-4922
}

\begin{abstract}
This study presents a novel approach for the early detection of developing thunderstorms. To date, methods for the detection of developing thunderstorms usually rely on accurate atmospheric motion vectors (AMVs) for the estimation of cooling rate of convective clouds, which corresponds to the updraft strength of the cloud objects. In this study we present a method for the estimation of the updraft strength that does not rely on AMVs. The updraft strength is derived directly from the satellite observations in the SEVIRI water vapor channels. For this purpose the absolute value of the vector product of two vectors derived from the observed radiances emitted by the water vapor channels in 2 subsequent satellite images is calculated. The $\mathrm{x}$ and $\mathrm{y}$ components of the vectors consists of the temporal change of the spatial brightness temperature gradients in $\mathrm{x}$ - and $\mathrm{y}$-direction, the $\mathrm{z}$ component consists of the temporal variation of the brightness temperature at pixel level. The absolute value of the vector product is referred to as normalized updraft strength (NUS). The performance of the method has been investigated for 2 summer periods in 2016 and 2017 by validation with lightning data. Values of the Critical Success Index (CSI) of about $66 \%$ for the 2016 period and $62 \%$ for the 2017 period demonstrate the good performance of the method. The POD values for the experiments with the highest CSI values are 90.3 for 2016 period and 87.6 for the 2017 period, respectively The corresponding FAR values are $28.2 \%$ (2016) and $32.5 \%$ (2017), respectively. In summary, the method has the potential to reduce the forecast lead time significantly, and can be quite useful in regions without a well maintained radar network.
\end{abstract}

Keywords: cumulonimbus; thunderstorms; stability filter; aviation

\section{Introduction}

Cumulonimbus clouds (Cbs), also referred to as thunderstorm clouds, are a dangerous weather phenomenon. Lightning, wind gusts and turbulence, hail and heavy precipitation, associated with $\mathrm{Cbs}$, result in serious damages and danger to life. Early and reliable prediction of $\mathrm{Cbs}$ is therefore essential for the initiation of appropriate protective measures, e.g. the selection of alternate flighting routes for aeroplanes, the temporary closure of airports, or general outdoor precaution measures.

The authors of [1] have been likely the first who discovered and discussed the physics of satellite based detection of Cbs. They found that the difference between the IR window channel and the water vapor channel can be used to detect $\mathrm{Cbs}$. The approach is used nowadays for the operational detection of Cbs e.g. [2,3]. More recently, it has been shown that mature $\mathrm{Cb}$ can be detected by satellites with relative high POD and low FAR, if the brightness temperature difference method according to [1] is applied to two water vapor channels $(7.3 \mu \mathrm{m}$ and $6.2 \mu \mathrm{m})$ in combination with NWP filtering [4].

Cbs originate from rapid vertical updraft of humid and warm air enforced by constraint forces caused e.g. by mountains, radiative heating or cold fronts. The life cycle of $\mathrm{Cbs}$ can be described by several stages which are illustrated in figure 1.

During the first stage cumulus and towering cumulus clouds occur. The next stage, the developing stage, is characterized by a fast cooling of the cloud top resulting from a strong updraft. This strong updraft leads to significant friction between the cloud particles which is a trigger for lightning, in particular in the mixed phase region [5]. The early developing phase is also referred to as convective 


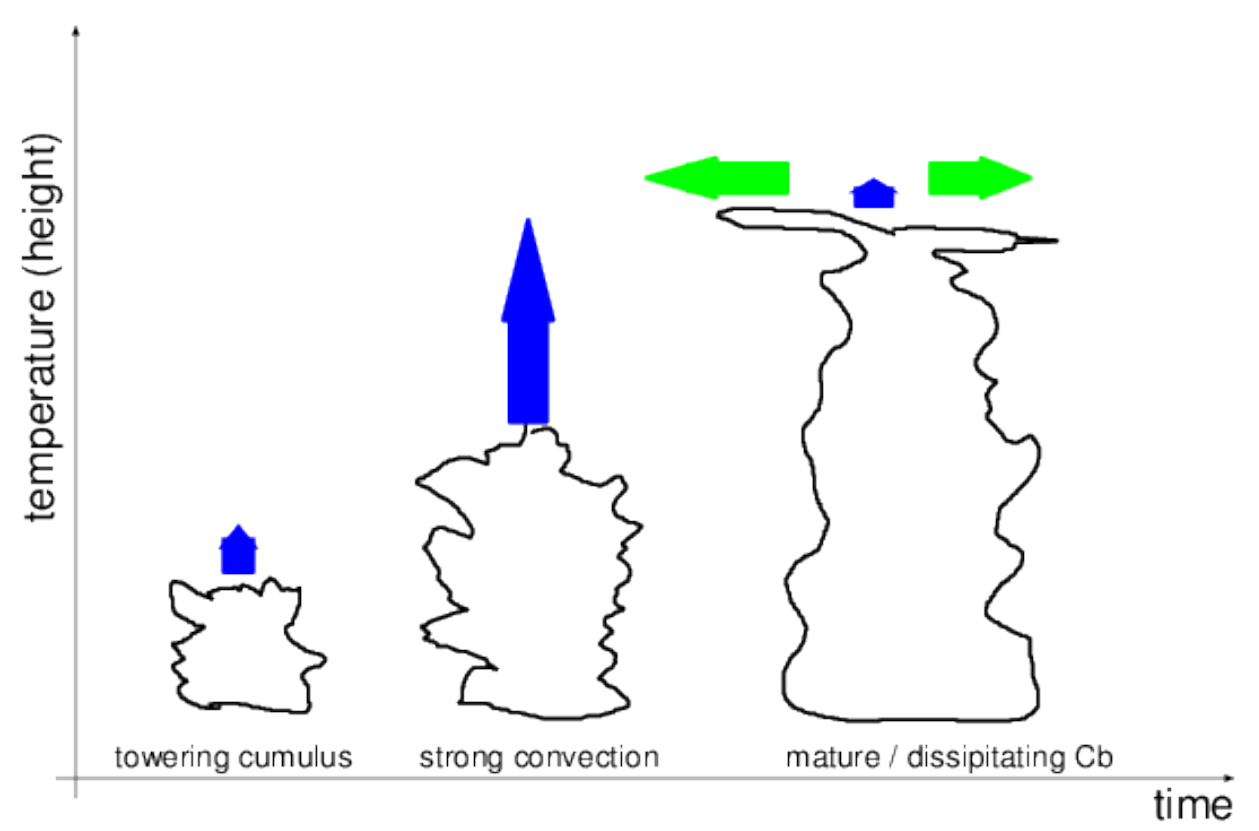

Figure 1. Illustration (simplified) of the generation of Cbs. Stage 1 towering cumulus, stage 2 strong convection and updraft of the cloud (developing thunderstorm) leading to the generation of a mature $\mathrm{Cb}$ (stage3). Once the $\mathrm{Cb}$ has reached the tropopause the updraft is retarded and kinetic updraft energy is transmitted into vertical movement/development. This leads to the development of an anvil and subsequent dissipation of the $\mathrm{Cb}$ after a certain lifetime.

initialization (CI). The updraft proceeds until the cell reaches the mature stage and dissipation starts. Mature cells can be accompanied by overshooting tops.

Thus, the strong vertical updraft of the clouds is theoretically a good indicator of developing thunderstorms and its reliable detection would allow to improve the forecast lead time. If the movement of the cloud object would be well known, then the strong vertical updraft could be observed as cooling rate, defined by the temporal change of the brightness temperature of the cells (cloud objects) between 2 subsequent satellite images. This has motivated several authors to use the temporal change of the brightness temperature as essential interest fields (indicator) for the detection of convective initiation / developing thunderstorms, e.g. [6-10]. However, the movement of the cells between two subsequent satellite images needs to be known well in order to derive the cooling rate accurately. In other words, accurate meso-scale atmospheric motion vectors (AMV) are required. Yet, this poses a serious challenge as e.g. discussed in [11]. The basic problem of AMV methods is that they are under-determined. Convective cloud movement is a meso-scale 3-d phenomenom, but only 2-d satellite images are available for the detection of AMVs. Thus, all approaches which use AMV or cloud motion fields (CMV) need to deal with this under-determination. Consequently, convection leads typically to serious errors in the AMV, see e.g. [11,12]. Further, smoothing and filtering by quality procedures hamper the accurate detection of the relevant meso-scale movement, see [11] for further details. Moreover, the cooling rate signal is significantly blurred by the relative large pixel size of the SEVIRI (Spinning enhanced visible and infrared imager) instrument in Central Europe, which is of about 15-20 km² for the IR channels. Based on the studies of [13-15] (and references therein) it can be assumed that the majority of the cells are smaller than the satellite pixel size during the early updraft/developing phase. This in turn leads to noise and blurring of the relevant information.

The above mentioned effects results in serious amount of missed detection and false alarms. This in turn constitutes a serious handicap for the operational usage. 
For this reason an alternative approach is discussed and evaluated in this study. This approach does not rely on atmospheric motion vectors, but the updraft strength is directly derived from the observations in the water vapor channels of the SEVIRI instrument on-board of MSG. First, we generate two vectors containing the temporal change of the spatial brightness temperature gradient in $\mathrm{x}$ and $\mathrm{y}$ direction of the water vapor channels as $\mathrm{x}$ and $\mathrm{y}$ components and the temporal change of the brightness temperature at pixel level as z-component of the vectors. For vector 1 we use channel WV1 $(7,3 \mu \mathrm{m})$ and for vector 2 channel WV2 $(6.2 \mu \mathrm{m})$. The vector product is then applied. As a result of the construction of the vectors the absolute value of the vector is a measure for the strength of the vertical updraft (development). Further details and explanation are provided in section 2. Thus, the updraft strength can be derived without the need of atmospheric motion vectors, but the vertical movement is directly deduced from the satellite observations of the WV1 and WV2 channel. This breaks the problem down to the generation of 1-d information from 2-d information, thus, aiming to resolve the problem of under-determination associated with classical atmospheric motion vector approaches.

The authors are not aware of any similar approach applied so far for the detection of the vertical updraft strength. Thus, we believe that this is a novel approach, which is of benefit for the improvement of the detection of developing thunderstorms. Further, as the approach does not rely on AMVs the implementation and operational maintenance is straightforward.

The term convective initialization is not well suited for validation purposes in consideration of the large satellite pixel size of the satellite. Thus, we prefer the term developing thunderstorms. It means, that the cells are still increasing, active, and not mature cells in terms of brightness temperature difference of the water vapor channels. This issue will be further discussed in section 4

Observational data plays a pivotal role for the accurate detection and short term forecast of developing thunderstorms due to the shortcomings of NWP models. Over ocean and a vast number of countries, which are not well equipped with precipitation radars, satellites are the only observational source for the detection of developing thunderstorms, e.g. see [16]. Furthermore, dry thunderstorms, which are a serious source for wildfires [17], can not be detected with weather radars.

\section{Materials and Methods}

\subsection{Method}

The brightness temperatures of the water vapor channels of the SEVIRI instrument on board of the Meteosat Second Generation satellites (MSG) are used for the calculation of the upward strength of the convective cells.

The satellite brightness temperatures are derived from the level 1.5 rectified image data of digital counts by application of the Eumetsat calibration coefficients and conversion method. More detailed information on MSG and the SEVIRI instrument are given in [18].

The potential of the WV channel for the $\mathrm{Cb}$ detection has been firstly discussed in [1] and adapted to 2 water vapor channels by e.g. [4]. Here, we present a novel approach for the detection of developing thunderstorms. For the following explanation it is important to consider that the signal received by the satellite consists of the radiation emitted by the cloud top and the clear sky radiation above the cloud top. The different channels and their clear sky weighting functions are shown in figure 2

In a first step a 3 component vector is generated from each of the 2 water vapor channels WV1 $(7.3 \mu \mathrm{m})$ and WV2 $(6 \times 3 \mu \mathrm{m})$. The components of the vectors are defined as follows. The temporal change of the spatial brightness temperature gradients in $\mathrm{x}$ and $\mathrm{y}$ direction of the WV1 and WV2 channel represents the $x$ and $y$ components of the vectors. The temporal change of the brightness temperatures at pixel level represents the z-components of the vectors. In order to get the temporal change information subsequent satellite images are used. The respective components are finally normalized by division of the BT in degrees Celsius of the respective channel. Please see equation 1 and equation 2 for the mathematical description of the vector components of $\mathbf{v b t f}_{7.3}$ and $\mathbf{v b t f}_{6.2}$. The components of the vectors can be interpreted as virtual brightness temperature fluxes (vbtf). 


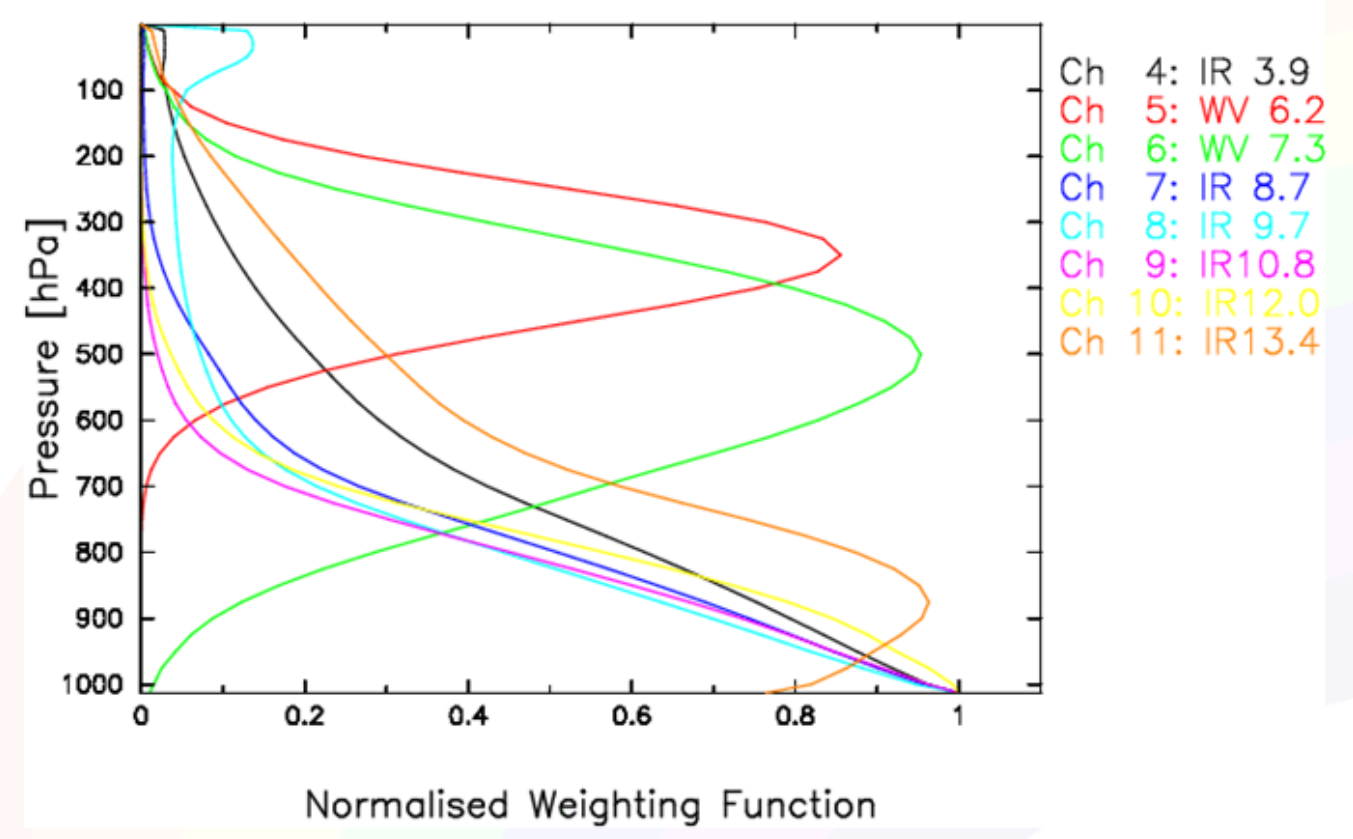

Figure 2. SEVIRI normalized weighting function of MSG for clear sky, source Eumetrain

$$
\begin{aligned}
v b t f_{x, 7.3} & =\frac{\delta}{\delta t \delta x} B T_{7.3}(t, x, y) /\left(B T_{7.3}(t o, x, y)-273\right) \\
v b t f_{y, 7.3} & =\frac{\delta}{\delta t \delta y} B T_{7.3}(t, x, y) /\left(B T_{7.3}(t o, x, y)-273\right) \\
v b t f_{z, 7.3} & =\frac{\delta}{\delta t}\left(B T_{7.3}(t, x, y) /\left(B T_{7.3}(t o, x, y)-273\right)\right. \\
v b t f_{x, 6.2} & =\frac{\delta}{\delta t \delta x} B T_{6.2}(t, x, y) /\left(B T_{6.2}(t o, x, y)-273\right) \\
v b t f_{y, 6.2} & =\frac{\delta}{\delta t \delta y} B T_{6.2}(t, x, y) /\left(B T_{6.2}(t o, x, y)-273\right) \\
v b t f_{z, 6.2} & =\frac{\delta}{\delta t}\left(B T_{6.2}(t, x, y) /\left(B T_{6.2}(t o, x, y)-273\right)\right.
\end{aligned}
$$

Then, the vector product of the 2 vectors are generated. The resulting vector is defined by the authors as the Normalized Updraft Strength (NUS) Vector

$$
\mathbf{N U S V}=\mathbf{v b t f}_{7.3} \times \mathbf{v b t f}_{6.2}
$$

The absolute value of NUS

$$
\text { NUS }=|\mathbf{N U S V}|
$$

which corresponds to

$$
N U S=\left|\mathbf{v b t f}_{7.3}\right| \cdot\left|\mathbf{v b t f}_{6.2}\right| \cdot \sin (\alpha)
$$

is a measure for the vertical updraft strength. This is explained in more detail in the subsequent paragraph. 
The movement of cloud and water vapor objects occurs in a 3 dimensional space. Thus, all components of the vectors $\mathbf{v b t f}_{7.3}$ and $\mathbf{v b t f} \mathbf{f}_{6.2}$ could hold contributions resulting from pure advection (horizontal movement) and convection (vertical movement / updraft.)

For explanation, let us assume that only horizontal movements of the cloud and water vapor objects do exist and that these objects do not change significantly in size or shape. The air is usually moist close to clouds. Thus, horizontal gradients between the clouds and the surrounding atmosphere are decreased by the radiation emitted by water vapor. This leads to the typical milky apparency of WV images in contrast to images of the IR window channel, in which the cloud borders sticks well out. In absence of vertical updraft, the horizontal gradients are relatively smooth and the temporal change of smooth spatial gradients is on average relative small. Strong convection (vertical updraft) enhances the spatial gradients significantly, as can be observed in the water vapor imagery. Further, it increases the $v b t f_{z}$ components significantly, which would be otherwise only be resulting from the small temporal change of the spatial gradients. In other words, absolute values of the vectors (equation 1 and 2) are on average lower for pure advection than for advection combined with a vertical updraft. Thus, $\left|\mathbf{v b t f}_{7.3}\right|$ and $\left|\mathbf{v b t f}_{6.2}\right|$ of equation 5 are on average higher if strong vertical updraft occurs.

Further, assuming again that only idealized horizontal movement occurs, then there is no change in the relation of the weighting function (clear sky) and the cloud top height along a pure horizontal movement. Thus, the relation of the radiation received by the WV2 channel and WV1 channel does not change along the movement. As the $v b t f_{z}$ components of WV1 and WV2 result only from the horizontal movement, there is no change along the movement in the relation between the $v b t f_{z}$ components and the horizontal components as well. In other words if only horizontal movement of rigid cloud objects is assumed, then the vectors $\mathbf{v b t f} 7.3$ and $\mathbf{v b t f} 6.2$ have a constant relation along the movement and they can be assumed to be parallel. Yet, the absolute value of a vector product of 2 parallel vectors is simply zero. Thus, for pure horizontal movement of rigid cloud objects the $\sin (\alpha)$ in equation 5 tends towards zero. Contrarily, if a vertical movement (updraft) occurs, than the vectors are no longer parallel and NUS would be notably greater than 0 . This results from the fact that the relation between the weighting functions as well as the cloud top height changes along a vertical movement of the cloud.

In summary, pure horizontal movement of rigid cloud objects leads on average to low values of $\left|\mathbf{v b t f}_{7.3}\right|$ and $\left|\mathbf{v b t f}_{6.2}\right|$ and $\sin (\alpha)$ tends towards zero, in contrast to vertical updraft. Thus, we postulate that the vector product, defined in equation 3 , can only be significantly above zero if a significant vertical updraft does exist. The validation will proof if this hypothesis is suited to detect developing thunderstorms.

Theoretically, we could assume that the approach works fine. However, in reality we are not dealing with mathematical derivations but are restricted to the satellite pixel resolution, meaning $\delta x$, $\delta y$ is a at least a 1-pixel shift in $\mathrm{x}$ (column) or $\mathrm{y}$ (line) direction respectively, and, $\delta t$ is a 15 minute time slot. Further, the SEVIRI specification for the calibration uncertainty is $0.75 \mathrm{~K}$ at $250 \mathrm{~K}$ for WV1 and WV2 [19] Assuming uncorrelated variables this induces additional significant noise for the calculation of NUS. Thus, the signal is blurred and noisy. Finally, updrafts are not exclusively correlated with (linked to) a $\mathrm{Cb}$. These items will be further discussed in section 4 .

In accordance with the evidences given in [4] a NWP stability filter is used to reduce the false alarm rate and to increase the CSI. For the stability filters the well established convective available potential energy (CAPE) [20] and as supplementing filter the totals (TT) total index (TT) are used, according to [4]. The thresholds are 60 and 50 respectively. Thus, with the NWP filter switched on, developing thunderstorms are only allowed if CAPE exceed a value of 60 or TT a value of 50 . The aim is to reduce false alarms where clouds develop / arise without evolving into thunderstorms. The thresholds are very low cause only stable and neutral atmospheres are aimed to be excluded. Please see sections 3 and 4 as well as [4] for further discussions and details.

For CAPE and TT the forecast runs of the ECMWF operational model [21] at $00 \mathrm{~h}$ and $12 \mathrm{~h}$ has been used. This means that forecasts up to $9 \mathrm{~h}$ are applied. A detailed documentation of the model can be found at the ECMWF web-page, see [22] Further, details and discussions are given in [4]. 


\subsection{Validation approach}

If lightning occurs the cloud is by convention a $\mathrm{Cb}$. Thus, $\mathrm{Cbs}$ can be validated by the use of lightning data, e.g. [4]. As developing thunderstorms evolve into mature Cbs they are expected to produce lightning at a certain point of their developing phase. Thus, they are expected to show lightning activity after the satellite image has been received. Thus, we use lightning data subsequent to the date of the satellite scan. Only lightning that occurs several minutes after the scanning time are considered for the validation. In this manner lightning data can be used to evaluate if the normalized updraft strength NUS, defined in 3, is a reasonable indicator of developing thunderstorms.

Lightning data from a low frequency (VLF/LF) lightning detection network (LINET), discussed in [23] and [24], are used for validation. The study focuses on Central Europe where the LINET network density is highest, see Figure 3. The borders of the validation region are as follows: Latitude 45.5N-56.5N and Longitude 2.0E-18.0E. The skill scores Probability Of Detection (POD), False Alarm Rate (FAR), Critical Success Index (CSI) and the accuracy (ACC) are used to measure the performance of the different experiments. For completeness the quantities are defined below:

$$
\begin{gathered}
\mathrm{POD}=\mathrm{CD} /(\mathrm{CD}+\mathrm{MD}) \\
\mathrm{FAR}=\mathrm{FD} /(\mathrm{CD}+\mathrm{FD}) \\
\mathrm{CSI}=\mathrm{CD} /(\mathrm{CD}+\mathrm{MD}+\mathrm{FD}) \\
\mathrm{ACC}=(\mathrm{CD}+\mathrm{CDN}) /(\mathrm{CD}+\mathrm{MD}+\mathrm{FD}+\mathrm{CDN})
\end{gathered}
$$

Hereby, CD stands for Correct Detection of a Cb, MD stands for Missed Detection of a $\mathrm{Cb}$ and FD stands for False Detection and CDN stands for Correct Detection of nil. The validation is performed on a pixel basis. This means that each pixel is assigned with either CD, FD, MD or CDN. CD, FD and MD are defined according to the criteria mentioned below.

A developing thunderstorm is defined as correctly detected if lightning occurs within a $15 \mathrm{~min}$ (25 $\mathrm{min}$ ) time interval after the satellite image is completely received and within a search region (SR) of $\pm 32 \mathrm{~km}$. The means only lightning in the time interval + 4-21 (+4-31) minutes are considered in the main experiments, as the scanning time of central Europe is approximately 3-4 minutes before the data is completely received. If there is no lightning, then a false alarm is counted. Missed detection is counted if lightning occurs but NUS is below the threshold within the search region. The search region (SR) of $\pm 32 \mathrm{~km}$ accounts for the typical range of dislocations between lightning and the center of the $\mathrm{Cb}$ (assumed to be approx. up to 15-20 km), as well as for the geolocation error of the satellite (approx $5 \mathrm{~km}$ ), for the movement of the cloud within the 15 minute interval and the uncertainty in the parallax correction.

In this study we focus on developing thunderstorms. For these reason all regions where the brightness temperature difference of the WV channel exceeds -1 degree are excluded from the validation. -1 degree constitutes the threshold for the detection of mature $\mathrm{Cbs}$ as defined and discussed in [4]. The exclusion of regions where mature Cbs are likely to occur is done to avoid the systematic distortion of the validation results. However, in order to investigate if the majority of detected cells are relative warm the above mentioned threshold has varied in some experiments, see section 3 for further details .

All experiments have been performed for 6, 9, 12, 15, 18 UTC respectively. The experiments have been done for the period 10th of May 2016 to 9th of June 2016 and June 2017 as in [4].

Both periods are characterized by frequent occurrence of thunderstorms for different weather situations. For the skill scores statistically significant populations have been examined. E.g., for the experiments with the optimal NUS threshold 8651 pixel are counted as hits, 3373 as false alarms and 927 as missed for the period in 2016 and for the 2017 period, 5966 are counted as hits, 2875 as false alarms and 843 as missed. 


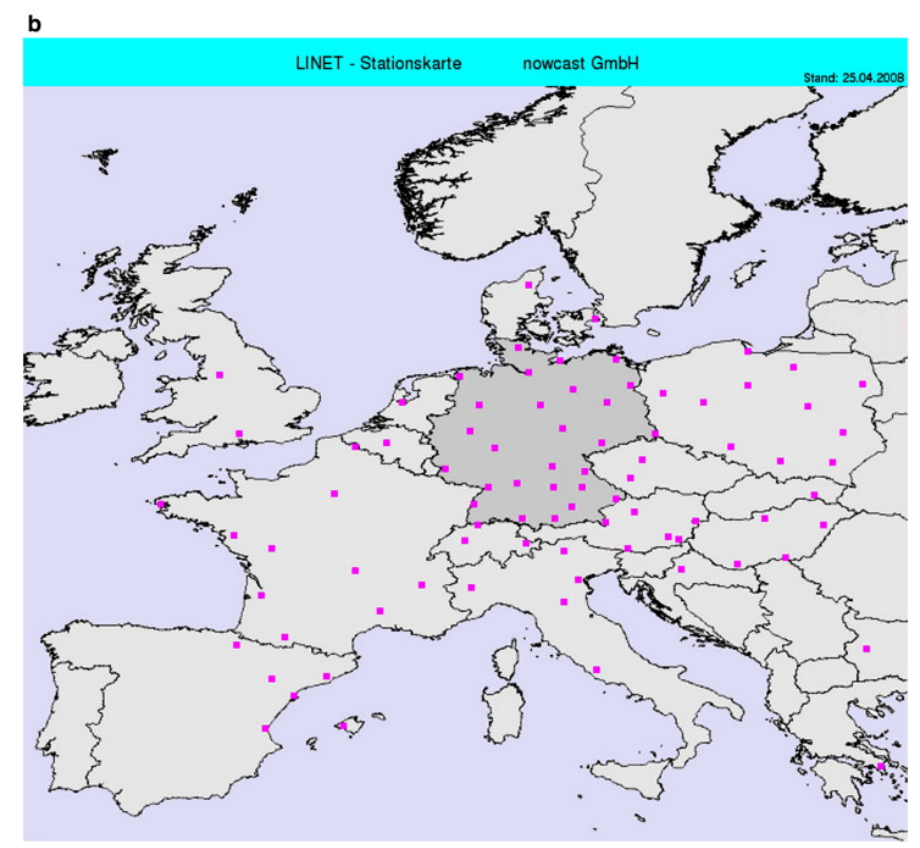

Figure 3. The LINET network, source LINET GMBH, taken from [23].

It is important to note that the skill scores are dedicated for the inter-comparison of the performed experiments within the scope of this study. In general, a comparison of skill scores is only reasonable for identical periods, regions, reference data and validation settings (e.g search region, pixel or object based validation,...). Thus, the skill scores presented in this manuscript are by no means representative for comparison with other methods.

\section{Results}

Figure 4 as well as Table 1 show the skill scores of the experiments. Figure 4 shows the results for variation of the NUS threshold for otherwise fixed experimental settings. Table 1 shows the results for various experimental settings, e.g. for prolonged time durations for the lightning data and experiments without NWP filtering. In the following the main results of the experiments are summarized.

The results provide clear evidence that the method works well. By consideration of the relative small size of developing thunderstorms and the relative large pixel size in Central Europe CSI values of about $66 \%$ (2016) and $63 \%$ (2017) are quite good, see Figure 4 and table 1 . While POD and FAR varies with the threshold settings CSI keeps quite stable for NUS ranging between $0.015-0.025$. This is a very good sign as it demonstrates the stability of the approach. Further, it allows the forecaster to select the optimal relation of POD and FAR for the respective application without losing significantly on CSI accuracy. 

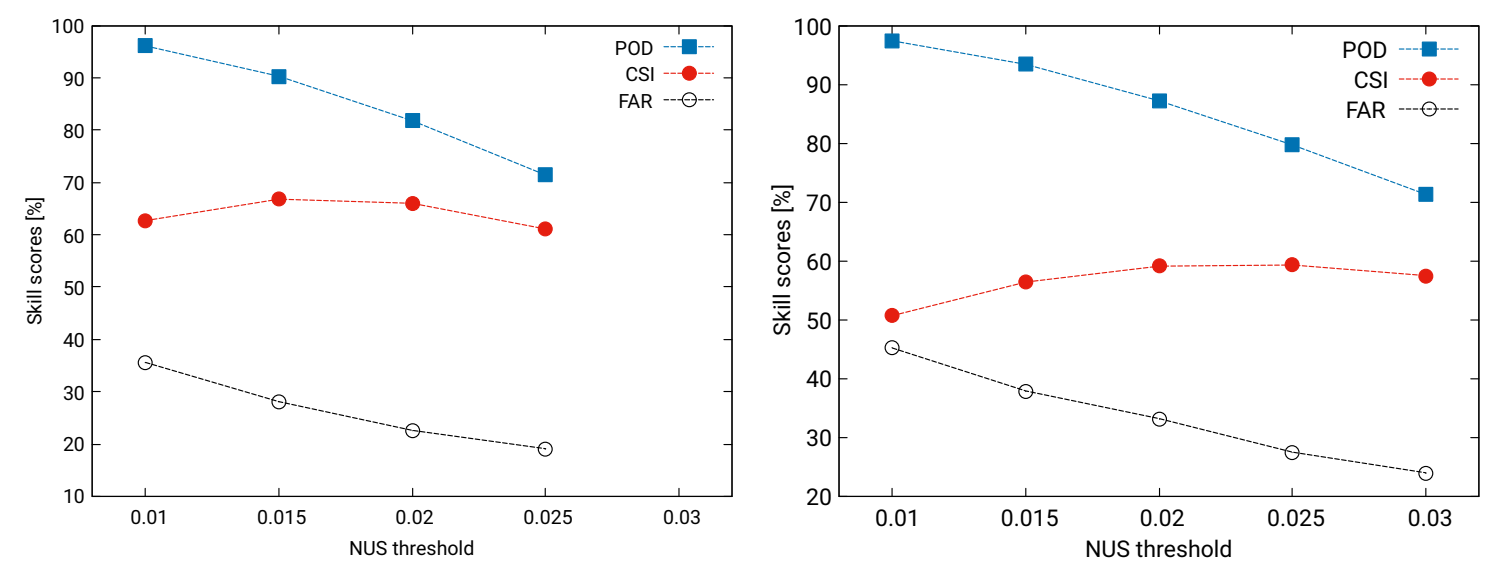

Figure 4. Skill scores (POD, FAR, CSI) in \% plotted against the NUS threshold for the 2016 (left) and 2017 (right) period and a SR of $35 \mathrm{~km}$, respectively. Left: POD and FAR decrease with increasing NUS. CSI is relative stable, but has the highest values around 0.015 for 2016 and 0.02 for 2017. For these experiments, the NWP filtering and the lightning settings have been according to that of experiment 1 in table 1

As in [4] the NWP filter does significantly increase the CSI. For the optimal NUS threshold the CSI values drops from 66.0 to 63.8 for 2016 and from 59.1 to 50.0 in 2017 without NWP filtering (Experiments 1,2 and 5,6 in Table 1). POD is increased without NWP filtering, but FAR is increased far more leading to a decrease in CSI. This decrease is much more pronounced in 2017, beside other effects, by the occurrence of large cold fronts (please see section 4 for further details).

Experiments 3 (2016) and 5 (2017) in comparison to 4 and 6 show that the extension of the lightning period used for the validation from $+4-19$ to $+4-29$ minutes leads to an improvement for both periods, but this improvement is much more pronounced in 2017. The improvement in CSI results pre-dominantly from a reduction of FAR, indicating that a significant number of developing cells show lightning not earlier than 19 minutes after the satellite scan.

Table 1. Results of the different experiments. NUS means the normalized updraft strength as defined in equation 3. Numerical Weather Prediction (NWP)/period provides the model used for NWP filtering and whether the investigated summer period was in 2016 or 2017. IFS is the forecast model of ECMWF and ICON of DWD. The other columns provide the probability of detection (POD), false alarm rate (FAR) and the critical success index (CSI). CAPE $>60$ or TX $>50$

\begin{tabular}{ccccccc}
\hline $\mathbf{N r}$ & CI If & NWP/Period & POD(\%) & FAR(\%) & CSI(\%) & time lightning \\
\hline 1 & NUS $>0.02$ & IFS/2016 & 81.8 & 22.6 & 66.0 & $+4-19$ min \\
\hline 2 & NUS $>0.02$ & none $/ 2016$ & 85.9 & 28.6 & 63.8 & $+4-19 \mathrm{~min}$ \\
\hline 3 & NUS $>0.015$ & IFS $/ 2016$ & 90.3 & 28.1 & 66.8 & $+4-19 \mathrm{~min}$ \\
\hline 4 & NUS $>0.015$ & IFS/2016 & 88.6 & 25.1 & 68.3 & $+4-29 \mathrm{~min}$ \\
\hline 5 & NUS $>0.02$ & IFS $/ 2017$ I & 89.2 & 36.4 & 59.1 & $+4-19 \mathrm{~min}$ \\
\hline 6 & NUS $>0.02$ & none $/ 2017$ & 90.1 & 47.6 & 49.8 & $+4-19 \mathrm{~min}$ \\
\hline 7 & NUS $>0.02$ & IFS/2017 I & 87.6 & 32.5 & 61.6 & $+4-29 \mathrm{~min}$ \\
\hline \hline
\end{tabular}

We did also runs where all regions with Brightness Temperature (BT) difference greater than -3 and -5 respectively are excluded from the validation. It is important to consider that the BT difference of the water vapor channels (WV1-WV2) decreases with increasing cloud top temperature for the interpretation of these experiments (not shown in the table). Thus lower values of the BT difference means warmer cells. Please see [4] for further details. The experiments have been done for the optimal 
NUS according to figure 4 and NWP filtering. In $201672 \%$ ( $85 \%$ ) of the correctly detected cells are below -5 degree (- 3 degree) BT difference, respectively. The CSI is only $2.8 \%(1.6 \%)$ lower and thus still above $63 \%$. In $201778 \%$ (91\%) of the correctly detected cells are below -5 degree BT (- 3 degree BT) difference, respectively. The CSI is only $2.6 \%$ (1.5\%) lower. The results show that the majority of the cells are relative warm, proofing that the majority of the cells are detected in a relative early developing phase.

We also investigated the effect of a smaller SR of .

Finally, we used lightning data from 2016 for the 2017 validation and vice versa for the optimal NUS values. This has been done to investigate the value of CSI resulting from pure chance for periods with relative high frequency of occurrence of thunderstorms. The CSI drops below $10 \%$ for both periods.

The accuracy is close to $100 \%$ for all experiments and is therefore not an appropriate skill score for $\mathrm{Cb}$ detection, although, it is sometimes used as score for events with low probability of occurrence.

\section{Discussion}

The results are very encouraging. However, the relative large FAR values should be discussed in more detail.

Clouds form usually in layers if the movement of the clouds is mainly horizontal. These are stratiform clouds and their movement should not cause serious problems for the method and should not significantly lead to False Alarm Rates. However, for cumulus like clouds air movement and development is mainly vertical. Thus, for these cloud types significant NUS values above 0 could occur. Yet, vertical updraft does not necessarily lead to a thunderstorm, e.g. cause of too low values of convective available potential energy (CAPE) or lack of additional significant lift induced by orography. These clouds are therefore likely a major reason for the false alarms. We would like to discuss this in more detail in order to clarify that a false alarm is not necessarily linked with an incorrect detection of a vertical updraft. The 6th of June 2017 is a very good example for the above mentioned arguments. At the 6 th of June a frontal system went through Germany during daytime. Low CAPE values around 60 $\mathrm{J} / \mathrm{kg}$ occurred along the front at $06 \mathrm{am}$.

A cold front is the transition zone of cold, dry stable air and warm unstable air mass. Frontal systems are known to be associated with gradual frontal lifting, causing the formation of cumulus clouds and Cbs. The frontal lifting leads to a significant updraft and thus to relative high NUS values, but not necessarily to the development of Cbs. Areas along a front with significant updraft but not any developing thunderstorms are leading to false alarms. This is illustrated by an example in Figure 5 .

The cold front at 6th of June 2917 has moved during the day from west to east across the validation region. Later on thunderstorms occurred, but at 6 am only high NUS values are apparent without lightning / thunderstorms. The NWP filtering depresses the false alarms along the cold front significantly. However, there are also regions where the NWP filtering fails. Here, it is obvious that significant vertical updraft do exist, but the conditions for the development of thunderstorms are otherwise not favorable, e.g. low CAPE environment. This in turn leads to false alarms, although the NUS information is reasonable. Similar effects occur also prior to the passing of the front. A large frontal systems passing Germany during the validation times have been also apparent at the 4th of June adding serious amounts of false alarms. In both cases the frontal gradient is apparent for every investigated time slot. However, even if no mature cells develop, it can be assumed that the updraft leads to meteorological significant convection combined with heavy rain,turbulence and wind gusts. Therefore, the registered false alarms may not necessarily be adequate in such cases. A calculation of the probability for the detection of developing thunderstorm cells rather than a yes/no decision might reduce the detected problems concerning false alarms in low CAPE environments if the output of an ensemble based NWP would be used as basis for the NWP filter.

Concerning false alarms and missed detection, the following arguments have also to be considered. Larger convective cells with lower updrafts can have the same or even higher satellite derived NUS 
values than smaller cells with larger updraft, caused by the large pixels size and the resulting blurring and smearing of the cells. Further, cells with lower updrafts can have the same or even higher satellite derived NUS if the are optimally located in a satellite pixel and not smeared over several cells. These effects are illustrated in figure 6 The reduction of NUS by blurring can be compensated by a decrease of the NUS threshold. However, this in turn leads to an increase of the false alarms, induced by the effects mentioned above, see figure 6.
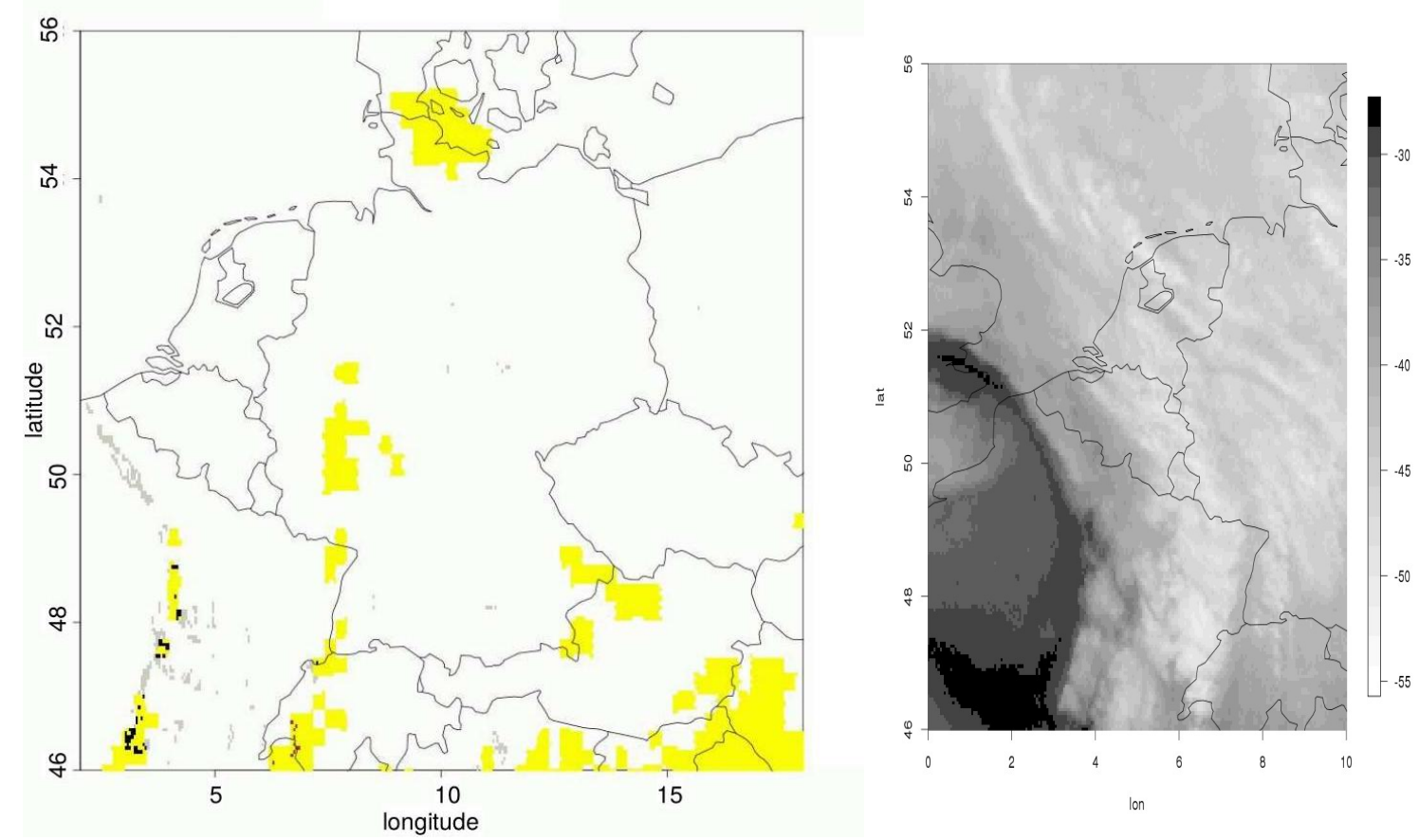

Figure 5. Example of the effect of false alarms due to a frontal system in a low CAPE environment (06.06.2017, 06 UTC). Right: The image of the water vapor channel. The frontal system is apparent in the WV channel as transition region with very dry (cloud free) and very moist (cloudy) air. Left: Image of the validation results. The red points are correctly detected developing thunderstorms, while the black points are examples for false alarms in a low CAPE environment. In grey are false alarms which are successfully filtered out. In yellow are all regions where the NWP filtering (CAPE $>60 \mathrm{~J} / \mathrm{kg}$ ) would allow the detection of developing thunderstorms. Depending on the temperature gradients and CAPE the frontal system could lead to the development of Cbs. However, in a low CAPE environment like in this case the frontal system leads only close to Switzerland to Cbs. The vertical lifting along the fronts leads to False Alarms (black color in the yellow regions) in regions where the NWP filtering fails. Obviously the number of false alarms are significant. This effect is also apparent at other days and times. 

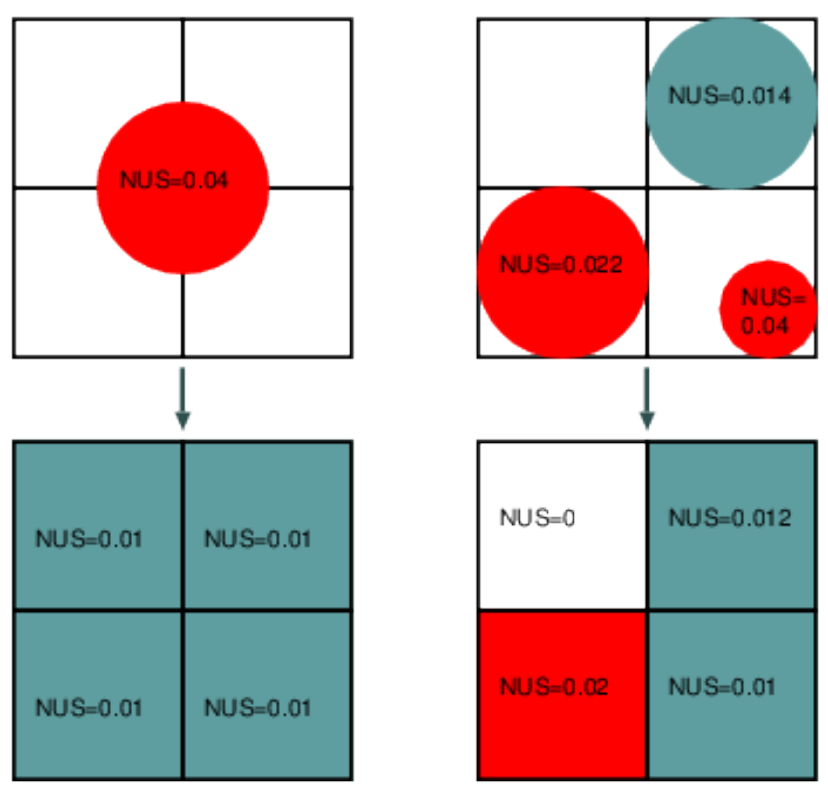

Real NUS values of the cells.

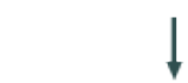

NUS values observed by satellite.

Figure 6. Illustration of the blurring effect on the observed NUS values. The objects colored in red stand for developing thunderstorms, the objects colored in grey stands for harmless clouds or missed detection cause of blurring. The values of the cell with a NUS values of 0.04 (left,top) would be observed in the relevant satellite pixels as 0.01 (left,bottom) cause of its location, which is caused by chance. Whereas the cell with 0.022 and 0.014 (right, top) would be observed close to their real values (right,bottom). Assuming a threshold of 0.15 the largest cell would not be seen and counted as Missed Detection. However, reducing the threshold to 0.1 would lead to an additional false alarm induced by the cell region with NUS of 0.12 and still the large cell would be counted as missed detection. Also the small cell with large updraft (right, top) would not be apparent in the satellite observations. Thus, increasing the NUS threshold decreases FAR, but decreases POD as well, and vice versa.

Further, as demonstrated in the validation, an extension of the duration for lightning decreases the false alarms but adds also additional missed detections. The start and duration of lightning can be quite different for developing thunderstorms. This effect adds artificial false alarms or artificial missed detection leading to lower CSI values. The CSI might be further improved by optimization of the NWP filtering, as demonstrated in [4].

The study has been performed based on the MSG prime Meteoat operational satellite imagery as it is dedicated for the operational use within aviation meteorology. Thus, the temporal resolution of subsequent images is 15 minutes. Convective cells with rapid cooling might be missed by the 15 minutes temporal resolution. It is likely that a higher temporal resolution, as e.g. planned for the prime MTG satellite, lead to an improvement of CSI. Yet, this will be further investigated after the launch of MTG, as this satellite comes with a significant higher spatial and temporal resolution.

\section{Conclusions}

The performed experiments show that, by consideration of the large pixel size, developing thunderstorm cells can be well detected by use of the normalized updraft strength (NUS). NUS is the absolute value of a vector product containing the temporal change of the horizontal brightness temperature (BT) gradients as $\mathrm{x}, \mathrm{y}$ components and the temporal change of BT at pixel level as z components. The respective components are calculated from subsequent images of the two SEVIRI water vapor channels. Values of the Critical Success Index (CSI) of about $66 \%$ for the 2016 period and $62 \%$ for the 2017 period demonstrate the good performance of the NUS method. The POD values for the highest CSI values of the experiments are with 90.3 (2016) and 87.6 (2017) quite high. But, remarkably they are accompanied by relative low FAR values, 28.2 for the 2016 period and 32.5 for 
the 2017 period. In particular, if the large satellite pixel size, causing smearing, blurring and noise is considered for the interpretation of the FAR values. Further, clear evidence has been given that the majority of the detected thunderstorm cells are warm, demonstrating that the cells are in an relative early developing phase. In summary, the method can be applied to reduce the forecast lead time significantly, in particular in regions without a well maintained radar network.

NWP filtering, according to [4], increases the CSI by $~ 2 \%$ in 2016 and $~ 9.5 \%$ in 2017. This indicates that NWP filtering is also of benefit for the early developing phase of thunderstorms.

More over, the results indicates that the method will be significantly gain on accuracy with the launch of Meteosat Third Generation, cause the blurring effects would be significantly reduced due to the great improvement in the spatial resolution of the imager on MTG.

Finally, the results show that the introduction of the concept of virtual brightness temperature fluxes is a promising way forward.

Acknowledgments: We thank the expert forecasters of DWD, in particular H. Koppert, A. Diehl and A. Barleben for the discussion and advice concerning the NWP stability filters. Many thanks to Kathrin Wapler for the discussions and support concerning lightning data.

Author Contributions: Richard Mueller developed the method and performed the validation study supported by Stephane Haussler. Matthias Jerg initialised and managed the project and contributed to the writing of the manuscript. Dirk Heizenreder is responsible for the overall development strategy and contributed to the writing of the manuscript as well.

Conflicts of Interest: The authors declare no conflict of interest.

\section{Abbreviations}

The following abbreviations are used in this manuscript:

$\begin{array}{ll}\text { ACC } & \text { Accuracy or hit rate } \\ \text { BT } & \text { Brightness Temperature } \\ \text { Cb } & \text { Cumulonimbus } \\ \text { CSI } & \text { Critical Success Index } \\ \text { CTH } & \text { Cloud Top Height } \\ \text { ECMWF } & \text { European Centre for Medium Weather forecast. } \\ \text { FAR } & \text { False Alarm Rate } \\ \text { ICON } & \text { NWP model of Deutscher Wetterdienst } \\ \text { KO } & \text { Convection Indec } \\ \text { MSG } & \text { Meteosat Second Generation } \\ \text { Meteosat } & \text { Meteorological satellite } \\ \text { NWP } & \text { Numerical Weather Prediction } \\ \text { POD } & \text { Probability of Detection } \\ \text { SEVIRI } & \text { Spinning enhanced visible and infrared imager } \\ \text { SR } & \text { Search Region }\end{array}$

1. Schmetz, J.; Tjemkes, A.; Gube, M.; van der Berg, L. Monitoring deep convection and convective overshooting with Meteosat. Advances in Space Research 1997, 19, 433-441.

2. Mosher, F. Detection of deep convection around the globe. Preprints, 10th Conf. on Aviation, Range, and Aerospace Me- teorology. American Meteorological Society, 2002, pp. 289-292. Portland.

3. Donovan, M.F.; Williams, E.R.; Kessinger, C.; Blackburn, G.; Herzegh, P.H.; Bankert, R.L.; Miller, S.; F., M. The Identification and VErificantion of Hazardous Convective Cells over Oceans Using Visible and Infrared Satellite Observations. Journal of Applied Meteorology and Climatology 2008, 47.

4. Müller, R.; Haussler, S.; Jerg, M. The Role of NWP Filter for the Satellite Based Detection of Cumulonimbus Clouds. Remote Sensing 2018, 10. https:/ / doi.org/10.3390/rs10030386.

5. Deierling, W.; Petersen, W.A.; Latham, J.; Ellis, S.; Christian, H.J. The relationship between lightning activity and ice fluxes in thunderstorms. Journal of Geographical Research 2008, 113. 
6. Mecikalski, J.; MacKenzie, W.; Koenig, M.; Muller, S. Cloud-Top Properties of Growing Cumulus prior to Convective Initiation as Measured by Meteosat Second Generation. Part I: Infrared Fields. JOURNAL OF APPLIED METEOROLOGY AND CLIMATOLOGY 2010, 49, 521-534.

7. Merk, D.and Zinner, T. Detection of convective initiation using Meteosat SEVIRI: implementation in and verification with the tracking and nowcasting algorithm Cb-TRAM. Atmos. Meas. Tech. 2013, 6, 1903-1918.

8. Okabe, I.; Imai, T.; IZUMIKAWA, Y. Detection of Rapidly Developing Cumulus Areas through MTSAT Rapid Scan Operation Observations. METEOROLOGICAL SATELLITE CENTER TECHNICAL NOTE 2011.

9. Autones, F. Algorithm Theoretical Basis Document for Convection Products. Technical report, NWC-SAF, 2016.

10. Lee, S.; Han, H.; Im, J.; Jang, E.; Lee, M.I. Detection of deterministic and probabilistic convection initiation using Himawari-8 Advanced Himawari Imager data. Atmospheric Measurement Techniques 2017, 10, 1859-1874.

11. Bedka, K.M.; Mecikalski, J.R. Application of Satellite-Derived Atmospheric Motion Vectors for Estimating Mesoscale Flows. JOURNAL OF APPLIED METEOROLOGY 2005, 44, 1761-1772.

12. Urbich, I.; Benidx, J.; M"uller, R. A Novel Approach for the Short-Term Forecast of the Effective Cloud Albedo. Remote Sensing 2018, 10.

13. Sengupta, S.K.; Welch, R.M.; Navar, M.; Berendes, T.A.; Chen, D.W. Cumulus Cloud Field Morphology and Spatial Patterns Derived from High Spatial Resolution Landsat Imageery. Journal of Applied Meteorology 1990, 29, 1245-1267.

14. H., H.R.; Orit, A.; Ilan, K.; Graham, F.; B., K.A.; P., K.A.; Mikhail, O.; Erick, F.; Guy, D.; Lital, P.; Ricki, Y.; Qian, C. Characterization of cumulus cloud fields using trajectories in the center of gravity versus water mass phase space: 2. Aerosol effects on warm convective clouds. Journal of Geophysical Research: Atmospheres 2016, 121, 6356-6373, [https: / / agupubs.onlinelibrary.wiley.com/doi/pdf/10.1002/2015JD024193].

15. Trapp, R.J. Mesoscale-Convective Processes in the Atmosphere; Cambridge, 2013.

16. Gijben, M.; Coning, C. Using Satellite and Lightning Data to Track Rapidly Developing Thunderstorms in Data Sparse Regions. Atmospher 2017, 8 .

17. Rorig, M.; Bothwell, P. Predicting Dry Lightning Risk Nationwide. Fire Science Brief 2012, 149. www.firescience.gov.

18. Schmetz, J.; Pili, Tjemkes, P.S.; Just, D.; Kerkmann, J.; Rota, S.; Ratier, A. An introduction to Meteosat Second Generation (MSG). Bull. Am. Met. Soc. 2002, pp. 977-992.

19. https://www.wmo-sat.info/oscar/instruments/view/503. last vistied on 21 th of August 2018.

20. Moncrief, M.W.; Miller, M.J. The dynamics and simulation of tropical cumulonimbus and squall lines. $Q$.J. R. Meteorol. Soc. 1976, 120, 373-394.

21. Bechtold, P.; Köhler, M.; Jung, T.; Doblas-Reyes, F.; Leutbecher, M.; Rodwell, M.J.; Vitart, F.; Balsamo, G. Advances in simulating atmospheric variability with the ECMWF model: From synoptic to decadal time-scales. Quarterly Journal of the Royal Meteorological Society 2008, 134, 1337-1351.

22. www.ecmwf.int/en/forecasts/documentation-and-support/changes-ecmwf-model/ifs-documentation. last visit 10.09.2017.

23. Betz, H.D.; Schmidt, K.; Laroche, P.; Blanchet, P.; Oettinger, W.P.; Defer, E.; Dziewit, Z.; Konarski, J. LINET — An international lightning detection network in Europe. Atmospheric Research 2009, 91, 564 - 573.

24. Betz, H.; Schmidt, K.; Oettinger, W.; Montag, B. Cell-tracking with lightning data from LINET. Advances in Geoscience 2008, 17, 55-61. 